International Journal of Pharmaceutics \& Pharmacology

\title{
Review of Experimental Pharmacological Studies of Plants Used for Asthma Management in Africa
}

Geneviève A. N'guessan-Irié, Landry S. Kouakou, Thierry A. Djadji, Julie A. Kouassi, Etienne K. Effo and Gisèle N. Siransy-Kouakou

Laboratory of Pharmacology, Training and Research Unit of Pharmaceutical and Biological Sciences, Felix Houphouet-Boigny University, 01 Abidjan, Côte d'Ivoire, Africa

Article info

Received 26 July 2020

Revised 17 August 2020

Available Online 24 August 2020

*Corresponding author: Amenan Geneviève N'guessan-Irié, Laboratory of Pharmacology, Training and Research Unit of Pharmaceutical and Biological Sciences, Felix Houphouet-Boigny University

\begin{abstract}
Objectives: The aim was to contribute to the promotion of medicinal plants used in Africa for management of asthma by analyzing their experimental pharmacological evaluation data.

Methods: We carried out a systematic review of the literature based on the following research equations in English and in French: "médecine traditionnelle africaine »ET « asthma »; "African traditional medicine » AND « asthma » or « Medicine African traditional » AND «asthma»; « plantes médicinales »ET «asthma »ET « antispasmodique »; «medicinal plants » AND «asthma » AND «anti-spasmodic »; " plantes médicinales $» E T$ «asthma »ET « anti-inflammatoire »; «medicinal plants » AND « asthma AND « anti-inflammatory».

Results: The study revealed 184 medicinal plants used in Africa for the treatment of asthma, 34 of which showed pharmacological properties in favor of their use. In animal experiments, 18 of these plants exerted a spasmolytic effect, 22 an anti-inflammatory effect and 6 both effects. The spasmolytic activity study protocols were based primarily on inhibition of smooth airway muscle contraction, including trachea, induced by various spasmogenic agents. As for of the methods used to study anti-inflammatory activity, the majority of them consisted of tests for carrageenan-induced edema or for formalin-induced leg irritation.

Conclusion: Our results justify the need for standardization of experimental study protocols for the recovery of medicinal plants.
\end{abstract}

Keywords: Asthma; Medicinal plants; Spasmolytic; Anti-inflammatory; Africa

\section{Introduction}

Asthma is increasing in an African population with high population growth, with air pollution [1,2], while medical infrastructure remains inadequate or inaccessible [3]. Thus, medicinal plants, inexpensive and easily accessible, are sometimes a first resort. Following the recommendations of the World Health Organization for the development of traditional medicines on the basis of evidence of safety, efficacy and quality control [4], we considered working on the theme of highlighting effectiveness. Thus, in order to contribute to the valorization of anti-asthmatic medicinal plants, the aim of this study was to list the plants which are used as traditional anti-asthmatic remedies in Africa, identify those that have been the subject of pharmacological experimental studies that demonstrate their antispasmodic and/or antiinflammatory properties, and analyse the experimental methods used in these published works. 


\section{Materials and Methods}

Our review was structured in three main stages:

\section{Literature search based on the following:}

- Target population: plants found in Africa, used by African traditional medicine in the management of asthma.

- Research tools: Health Sciences and Biosciences theses, reference books, and Internet research using:

- Databases: PubMed / Medline, Hinari Health, Scopus

- Search engines: Google, Google scholar

- English and French Search equation: "médecine traditionnelle africaine » ET « asthma »

« African traditional medicine » AND « asthma » or « Medicine African traditional » AND «asthma »

« plantes médicinales » ET « asthma » ET « antispasmodique »

«medicinal plants » AND « asthma » AND « antispasmodic »

« plantes médicinales » ET « asthma » ET « antiinflammatoire »

«medicinal plants » AND « asthma » AND « antiinflammatory »

2. Selection of articles and works according to the following criteria:

- Languages: French, English
- Media: written (electronic and paper)

- Presentation: understandable and readable texts; simple and clear styles.

- Contents: related to plants used in African traditional medicine in the treatment of asthma and the evaluation of antispasmodic and/or anti-inflammatory activity of these plants mentioned either in the title, introduction, conclusion or summary.

- Scientific quality: journal articles, institutional reports, guidelines, theses, references.

\section{Processing of results}

The aim was to extract the data for synthesis, through the translation of English-French texts and vice versa on the one hand, and the reading and comparative analysis of articles dealing with the same theme in order to retain only relevant and similar data on the other.

\section{Results}

Inventory of traditional anti-asthmatic plants in Africa

Table I shows the plant species published as antiasthmatic medicinal plants in Africa. We have identified 184 species of traditional use in the management of asthma in Africa.

Table 1: Plants used in the management of asthma in Africa.

\begin{tabular}{|c|c|c|c|}
\hline S. No. & Species & Family & Used Parts \\
\hline 1 & Acacia albida Del. & Mimosaceae & Bark, leaves, roots \\
\hline 2 & Acacia nilotica $($ L.) Willd ex Delile & Mimosaceae & Leaves, seeds \\
\hline 3 & Acacia senegalensis (L.) Willd ex Del. & Mimosaceae & Latex \\
\hline 4 & Adansonia digitata (L.) & Bombaceae & Leaves \\
\hline 5 & Aerva lanata (L.) Schult Juss. Ex & Amaranthaceae & Leaves \\
\hline 6 & Adhatoda vasica (L.) & Acanthaceae & Nuts \\
\hline 7 & Aframomum laurentii Schum. & Zingiberaceae & Seeds, Fruits \\
\hline 8 & Aframomum melegueta (Roscoe) K. Schum. & Zingiberaceae & Seeds \\
\hline 9 & Agava americana (L.) Subsp. & Agavaceae & Leaves \\
\hline 10 & Ageratum conyzoides $(\mathrm{L})$. & Astéraceae & Whole plan \\
\hline 11 & Ajuga iva (L.) & Lamiaceae & Leaves \\
\hline 12 & Alchornea cordifolia (Schumach. Thonn.) Mull.Arg. & Euphorbiaceae & Leaves \\
\hline 13 & Allium cepa (L.) & Liliaceae & Bulbs \\
\hline 14 & Allium sativum (L.) & Liliaceae & Pods \\
\hline 15 & Aloe vera (L.) Burm.F. & Liliaceae & Latex \\
\hline
\end{tabular}


Citation: N'guessan-Irié GA, Kouakou LS, Djadji TA, et al. Review of Experimental Pharmacological Studies of Plants Used for Asthma Management in Africa. Int J Pharm Pharmacol 2020; 4: 146. doi: 10.31531/2581$\underline{3080.1000146}$

\begin{tabular}{|c|c|c|c|}
\hline 16 & Ammi visnaga (L.) Lamark & Apiaceae & Fruits \\
\hline 17 & Anacardium occidentale (L.) & Anacardiaceae & Fruits, leaves, bark \\
\hline 18 & Ananas communis $(\mathrm{L})$. & Broméliaceae & Peduncles \\
\hline 19 & Anchomanes giganteus (Engl.) & Araceae & Stem \\
\hline 20 & Arachis hypogaea (L.) & Fabaceae & Seeds \\
\hline 21 & Achras sapota (L.) & Sapotaceae & Leaves \\
\hline 22 & Argemone mexicana (L.) & Papaveraceae & Leaves \\
\hline 23 & Aristolochia longa $(\mathrm{L})$. & Aristolochiaceae & Seeds, leaves \\
\hline 24 & Asystasa gangetica (L.) & Acanthaceae & Seeds, leaves \\
\hline 25 & Azadirachta indica (L.) & Meliaceae & Leaves, bark \\
\hline 26 & Baissea multiflora A.D.C. & Apocynaceae & Leaves \\
\hline 27 & Balanites aegyptiaca (L.) Del. & Bananitaceae & $\begin{array}{c}\text { Bark, Fruits, } \\
\text { leaves }\end{array}$ \\
\hline 28 & Baphia nitida Lodd. & Fabaceae & Leaves \\
\hline 29 & Bidens pilosa (L.) & Astéraceae & Bark \\
\hline 30 & Boerhavia diffusa L. Sp. & Nyctaginaceae & Leaves \\
\hline 31 & Brassica juncea (L.) Czern & Brasicaceae & Seeds, leaves \\
\hline 32 & Brassica oleracea (L.) & Brassicaceae & Leaves \\
\hline 33 & Bridelia micrantha (Hochst.) Baill. & Phyllanthaceae & Roots, bark \\
\hline 34 & Bridelia ndellensis Beille. & Phyllanthaceae & Roots \\
\hline 35 & Brillantaisia patula T. Anders. & Acanthaceae & Leaves \\
\hline 36 & $\begin{array}{c}\text { Brugmansia suaveolens (Humb. Et Bonpl. ex Willd.) } \\
\text { Bercht. et J. Presl }\end{array}$ & Solanaceae & Leaves \\
\hline 37 & Calotropis procera (Aiton) W.T. & Asclepiadaceae & Roots \\
\hline 38 & Cannabis sativa (L.) & Cannabinaceae & Leaves \\
\hline 39 & Cannarium schweinfurthii Engl. & Burseraceae & $\begin{array}{l}\text { Leaves, Fruits, } \\
\text { bark }\end{array}$ \\
\hline 40 & Carapa procera D.C & Meliaceae & Seeds \\
\hline 41 & Carica papaya $(\mathrm{L})$. & Caricaceae & Leaves \\
\hline 42 & Cassia italica (Mill.) Spreng. & Cesalpiniaceae & Leaves \\
\hline 43 & Cassia occidentalis (L.) Sp. & Cesalpiniaceae & Leaves \\
\hline 44 & Casuarina equisetifolia (L.) & Cassuarinaceae & Stem \\
\hline 45 & Celtis integrifolia (Lam.) & Ulmaceae & Leaves \\
\hline 46 & Citrus aurantifolia $\mathrm{L} . \mathrm{Sp}$ & Rutaceae & Fruits, bark \\
\hline 47 & Chrozophora senegalensis (Lam.) & Euphorbiaceae & Leaves, bark \\
\hline 48 & Crataegus monogyna Jacq. & Rosaceae & Buds \\
\hline 49 & Cochlospermum tinctorium Perr. ex A. Rich & Cochlospermanaceae & Leaves, roots \\
\hline 50 & Cocos nucifera (L.) & Aracaceae & Bark \\
\hline 51 & Cola cordifolia (Cav.) R. Br. & Sterculiaceae & Leaves, roots \\
\hline 52 & Cola gigantea A. Chev. & Malvaceae & bark \\
\hline 53 & Cola nitida (Vent.) Schott et Endl. & Sterculiaceae & Nuts \\
\hline 54 & Combretum acculeatum Vent. & Combretaceae & Leaves \\
\hline 55 & Combretum glutinosum Perr.ex D.C. & Combretaceae & Leaves, bark \\
\hline 56 & Combretum micranthum G. Don. & Combretaceae & Leaves \\
\hline 57 & Commelina diffusa Burm. F. & Commelinaceae & Leaves \\
\hline
\end{tabular}


Citation: N'guessan-Irié GA, Kouakou LS, Djadji TA, et al. Review of Experimental Pharmacological Studies of Plants Used for Asthma Management in Africa. Int J Pharm Pharmacol 2020; 4: 146. doi: 10.31531/2581$\underline{3080.1000146}$

\begin{tabular}{|c|c|c|c|}
\hline 58 & Convolvulus arvensis (L.) & Convolvulaceae & Leaves \\
\hline 59 & Crescentia cujetes (L.) & Bignonaceae & Leaves \\
\hline 60 & Curcuma longa (L.) & Cucurbitaceae & Rhizomes, leaves \\
\hline 61 & Cymbopogon giganteus Chiov & Poaceae & Leaves \\
\hline 62 & Cymbopogon septratus DC. & Poaceae & Leaves \\
\hline 63 & Cyphostemma adenocaule Desc. & Vitaceae & Leaves \\
\hline 64 & Dacryodes edipis (G. Don) H.J. Lam & Burseraceae & Bark \\
\hline 65 & Datarium microcarpium Guill et Perr. & Cesalpiniaceae & Roots \\
\hline 66 & Datura stramonium (L.) & Solanaceae & Leaves, Flowers \\
\hline 67 & Desmodium adscendens (Sw.) DC. & Fabaceae & Leaves \\
\hline 68 & Desmodium tortuosum DC. & Fabaceae & Leaves \\
\hline 69 & Dichrostachys cinerea $($ L.) Wight et Arn & Fabaceae & Roots, leaves \\
\hline 70 & Diospyros mespiliformis Hochst.ex A. Rich. & Ebenaceae & Leaves \\
\hline 71 & Elaeis guineensis Jacq. & Anecaceae & Seeds \\
\hline 72 & Eucalyptus camaldulensis Dehnh. & Myrtaceae & Leaves \\
\hline 73 & Eucalyptus globulus & Myrtaceae & Leaves \\
\hline 74 & Eugenia caryophyllata & Myrtaceae & Cloves \\
\hline 75 & Euphorbia balsamifera Ait. & Euphorbiaceae & Roots \\
\hline 76 & Euphorbia hirta (L.) & Euphorbiaceae & Whole plant \\
\hline 77 & Euphorbia milii Des. Moul. & Euphorbiaceae & Leaves \\
\hline 78 & Ficus benghalensis (L.) & Moraceae & Leaves, bark \\
\hline 79 & Ficus exasperata Vahl. & Moraceae & Leaves, roots, bark \\
\hline 80 & Ficus iteophylla Miq. & Moraceae & Leaves \\
\hline 81 & Ficus sycomorus (L.) & Moraceae & Leaves \\
\hline 82 & Ficus vallis-choudae Del. & Moraceae & Fruits, bark \\
\hline 83 & Foeniculum vulgare P. (Mill.) & Apiaceae & Seeds \\
\hline 84 & Glycyrrhiza glabra (L.) & Fabaceae & Roots \\
\hline 85 & Gossypium barbadense (L.) & Malvaceae & Leaves \\
\hline 86 & Guiera senegalensis J.F. Gmel. & Combretaceae & Leaves \\
\hline 87 & Helianthus annuus (L.) & Asteraceae & Seeds \\
\hline 88 & Hymenocardia acida Tul. & Euphorbiaceae & Leaves \\
\hline 89 & Hyperium perforatum (L.) & Hypericineae & Flowering heads \\
\hline 90 & Icacina senegalensis A. Juss. & Icacinaceae & Leaves, roots \\
\hline 91 & Illicium verum Hook.F. & Schisandraceae & Fruits \\
\hline 92 & Jatropha curcus (L.) & Euphorbiaceae & Leaves \\
\hline 93 & Juniperus phoenicea (L.) & Cupressaceae & Flowering heads \\
\hline 94 & Kigelia africana (Lam.) Benth. & Bignoniaceae & Leaves \\
\hline 95 & Lannea acida A. Rich. & Anacardiaceae & Bark \\
\hline 96 & Lantana camara $\mathrm{L}$. & Verbenaceae & Leaves \\
\hline 97 & Lavandula angustifolia Mill & Lamiaceae & Flowering heads \\
\hline 98 & Lavandula multifida L. & Lamiaceae & Flowering heads \\
\hline 99 & Lavandula stoechas L. & Lamiaceae & Flowering heads \\
\hline 100 & Lawsonia inermis L. & Lythraceae & Leaves \\
\hline 101 & Lepidium sativum L. & Brassicaceae & Leaves, Fleurs \\
\hline
\end{tabular}


Citation: N'guessan-Irié GA, Kouakou LS, Djadji TA, et al. Review of Experimental Pharmacological Studies of Plants Used for Asthma Management in Africa. Int J Pharm Pharmacol 2020; 4: 146. doi: 10.31531/2581$\underline{3080.1000146}$

\begin{tabular}{|c|c|c|c|}
\hline 102 & Leptadenia hastata Decne & Asclepiadaceae & Leaves \\
\hline 103 & Linum usitatissimum L. & Linaceae & Seeds \\
\hline 104 & Lippia chevalieri L. & Verbenaceae & Leaves \\
\hline 105 & Lobelia inflata $\mathrm{L}$. & Campanulaceae & Leaves \\
\hline 106 & Lobelia siphilitica $\mathrm{L}$. & Campanulaceae & Roots \\
\hline 107 & Mammiophytum fulvum Mull & Euphorbiaceae & Leaves \\
\hline 108 & Mandragora autumnalis Bert & Solanaceae & Leaves \\
\hline 109 & Mangifera indica $\mathrm{L}$. & Anacardiaceae & Leaves \\
\hline 110 & Marrubium valgare L. & Lamiaceae & Leaves \\
\hline 111 & Melaleuca viridiflora Gaertner & Myrtaceae & Leaves \\
\hline 112 & Melissa officinalis L. & Lamiaceae & Aerial parts \\
\hline 113 & Mentha piperata $\mathrm{L}$. & Lamiaceae & Aerial parts \\
\hline 114 & Mentha pulegium $\mathrm{L}$. & Lamiaceae & Aerial parts \\
\hline 115 & Mentha spicata L. & Lamiaceae & Leaves \\
\hline 116 & Microgrossa pyrifolia Lam & Asteraceae & Fruits \\
\hline 117 & Morinda lucida Benth & Rubiaceae & Leaves \\
\hline 118 & Morinda morindoides Milue & Rubiaceae & Leaves \\
\hline 119 & Moringa oleifera Lam & Moringaceae & $\begin{array}{c}\text { Leaves, seeds, } \\
\text { roots }\end{array}$ \\
\hline 120 & Musa acuminata Cola & Musaceae & Leaves \\
\hline 121 & Musanga cecropioides $\mathrm{R}$. Br. & Moraceae & Leaves \\
\hline 122 & Myrianthus arboreus $\mathrm{P}$ & Moraceae & Root bark \\
\hline 123 & Myrtus communis $\mathrm{L}$. & Myrtaceae & $\begin{array}{c}\text { Seeds, Fruits, } \\
\text { leaves }\end{array}$ \\
\hline 124 & Nerium oleander L. & Apocynaceae & Leaves \\
\hline 125 & Nicotiana rustica $\mathrm{L}$. & Solanaceae & Leaves \\
\hline 126 & Nigella sativa $\mathrm{L}$. & Ranunculaceae & Seeds \\
\hline 127 & Ocimum bassilicum $\mathrm{L}$. & Lamiaceae & $\begin{array}{c}\text { Leaves, whole } \\
\text { plant }\end{array}$ \\
\hline 128 & Oxythenanthera abyssinica Munro & Poaceae & Leaves \\
\hline 129 & Parinari macrophylla Sabine & Rosaceae & $\begin{array}{c}\text { Leaves, Fruits, } \\
\text { roots }\end{array}$ \\
\hline 130 & Pakia biglobosa Jacq. & Mimosaceae & Leaves \\
\hline 131 & Palisota ambigua $\mathrm{L}$. & Commelinaceae & Whole plant \\
\hline 132 & Pennisetum panicum L. & Poaceae & Leaves \\
\hline 133 & Pennisetum thyphoides Stapf et Hubb & Gramineae & Seeds \\
\hline 134 & Persea americana $\mathrm{L}$. & Lauraceae & Leaves \\
\hline 135 & Petroselinum sativum $\mathrm{L}$. & Ombelifereae & Leaves \\
\hline 136 & Phyllanthus acidus L. & Euphorbiaceae & Leaves, Fruits \\
\hline 137 & Piliostigma reticulatum DC & Cesalpiniaceae & Leaves, bark \\
\hline 138 & Piper betle L. & Piperaceae & Leaves \\
\hline 139 & Piper guineensis Schum. \&Thonn. & Piperaceae & Leaves \\
\hline 140 & Piper longum L. & Piperaceae & Fruits \\
\hline 141 & Pistacia lentiscus $\mathrm{L}$. & Anacardiaceae & Stem resins \\
\hline 142 & Platycerium stermarie DC & Polypodiaceae & Bark \\
\hline 143 & Plumbago zeypanica L. & Plumbaginaceae & Leaves \\
\hline
\end{tabular}


Citation: N'guessan-Irié GA, Kouakou LS, Djadji TA, et al. Review of Experimental Pharmacological Studies of Plants Used for Asthma Management in Africa. Int J Pharm Pharmacol 2020; 4: 146. doi: 10.31531/2581$\underline{3080.1000146}$

\begin{tabular}{|c|c|c|c|}
\hline 144 & Prosopis africana Taub & Cesalpiniaceae & Roots, leaves \\
\hline 145 & Prunus cerasus L. & Rosaceae & Fruits \\
\hline 146 & Pseudo mussaenda stenocarpa (Hiern.) Petit & Rubiaceae & Leaves \\
\hline 147 & Psidium guajava $\mathrm{L}$. & Myrtaceae & Leaves \\
\hline 148 & Punica granatum $\mathrm{L}$. & Pinicaceae & Leaves \\
\hline 149 & Pupalia lappacea $\mathrm{L}$. & Amaranthaceae & Fruits \\
\hline 150 & Ricinus communis $\mathrm{L}$. & Euphorbiaceae & Leaves \\
\hline 151 & Rosmarinus officinalis L. & Lamiaceae & Whole plant \\
\hline 152 & Saba senegalensis Pichon & Apocynaceae & Leaves \\
\hline 153 & Salvadora persica $\mathrm{L}$. & Salvadoraceae & Leaves, roots \\
\hline 154 & Salvia officinalis L. & Lamiaceae & Leaves \\
\hline 155 & Sanguisorba minor Scop & Rosaceae & Leaves \\
\hline 156 & Scelerocacya birrea A. & Anacardiaceae & $\begin{array}{c}\text { Bark, Fruits, } \\
\text { Fleurs }\end{array}$ \\
\hline 157 & Scyphocephalium ochocoa Warb & Myristicaceae & Bark, leaves \\
\hline 158 & Sesamum indicum $\mathrm{L}$. & Pedeliaceae & Leaves \\
\hline 159 & Smilax aspera $\mathrm{L}$. & Smilacaceae & Roots, Fruits \\
\hline 160 & Solamum melongena L. & Solanaceae & Fruits \\
\hline 161 & Sterculia stegera Del. & Sterculiaceae & Bark \\
\hline 162 & Sterculia tragacantha Lind $\mathrm{P}$ & Sterculiaceae & $\begin{array}{c}\text { Bark, leaves, } \\
\text { Fruits }\end{array}$ \\
\hline 163 & Tamarindus indica $\mathrm{L}$. & Caesalpiniaceae & $\begin{array}{c}\text { Fruits, roots, } \\
\text { leaves }\end{array}$ \\
\hline 164 & Tapinanthus bangwensis Engl. & Loranthaceae & Leaves \\
\hline 165 & Tectrorchidium didymostemon Baill & Euphorbiaceae & Stem bark \\
\hline 166 & Terminalia avicennö̈des $\mathrm{G}$ et Perr & Combretaceae & Leaves \\
\hline 167 & Terminalia catappa L. & Combretaceae & Leaves \\
\hline 168 & Terminalia chebula $\mathrm{RTZ}$ & Combretaceae & Fruits, roots \\
\hline 169 & Terminalia macroptera Gill et $\mathrm{P}$ & Combretaceae & Leaves \\
\hline 170 & Thormandersia hensii DE Will & Acanthaceae & Leaves \\
\hline 171 & Thuya orientalis L. & Cupressaceae & Leaves \\
\hline 172 & Thymus vulgaris $\mathrm{L}$. & Lamiaceae & Leaves \\
\hline 173 & Trigonella foenum graecum $\mathrm{L}$. & Apiaceae & Seeds \\
\hline 174 & Tylophora sylvation Decno & Apocynaceae & Root bark \\
\hline 175 & Vernonia caferta Benth & Asteraceae & Stem bark \\
\hline 176 & Vernonia colorata (Willd) Drake & Asteraceae & Leaves \\
\hline 177 & Viscum album $\mathrm{L}$. & Santalaceae & Leaves \\
\hline 178 & Vitex doniana Sweet & Verbenaceae & Leaves \\
\hline 179 & Waltheria indica $\mathrm{L}$. & Malvaceae & Whole plant \\
\hline 180 & Ximenia americana $\mathrm{L}$. & Olaaceae & Leaves \\
\hline 181 & Xylopia aethiopica A. Rich & Annonaceae & Seed \\
\hline 182 & Zea mays L. & Poaceae & Stigmata \\
\hline 183 & Zingiber officinal Roscoe & Zingiberaceae & Rhizomes \\
\hline 184 & Ziphyphus mauritiana Lam & Rhamnaceae & Leaves \\
\hline
\end{tabular}




\section{Pharmacological properties demonstrated in animal experiments}

In preclinical experiments, 34 plants showed properties against bronchospasm and/or inflammation, including 18 for antispasmodic property, 22 for anti-inflammatory property and 6 for both pharmacological properties (Figure 1).

Experimental protocols for the demonstration of pharmacological properties

In vivo, ex vivo and in vitro tests were used in the protocols for the study of anti-asthmatic activity. The bronchospasm-related aspect of asthma was explored through 14 tests, including 4 whole animal tests [5-8] and 10 isolated organs, including the trachea and to a lesser extent the intestine [9-18] as shown in Table 2.
The inflammatory component was evaluated using 17 tests including 11 in vivo [19-28;5] and 6 in vitro $[17,29,20,30-32]$ according to the data in Table 3 . The frequency of implementation of testing on biological materials is shown in Figure 2.

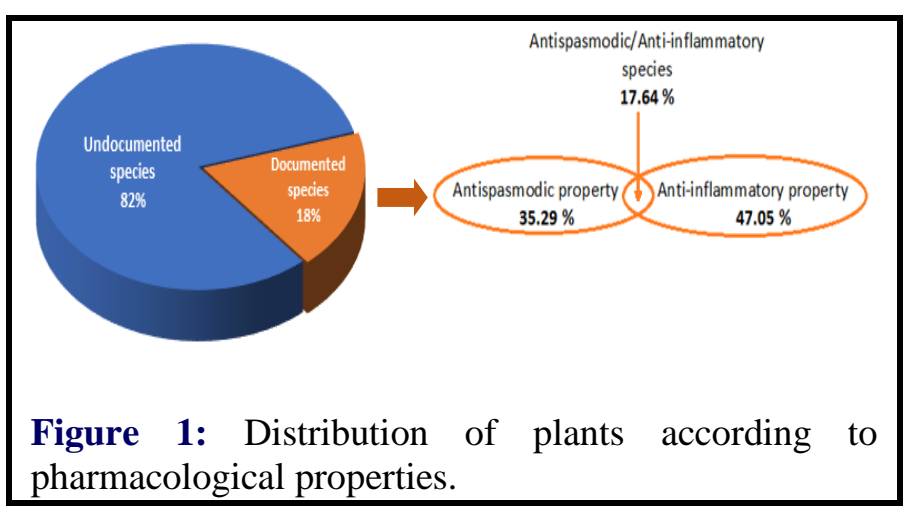

Table 2: Experimental protocols for highlighting antispasmodic activity.

\begin{tabular}{|c|c|c|c|}
\hline Methods & Plants & Results & References \\
\hline \multicolumn{4}{|l|}{ In vivo } \\
\hline $\begin{array}{c}\text { Oral administration of plant extract } \\
\text { in pre-treatment, then exposure to } \\
\text { the mast cell allergen. }\end{array}$ & Adhatoda vasica & $\begin{array}{c}\text { Inhibition of mast cell } \\
\text { degranulation. }\end{array}$ & [5] \\
\hline Oral administration of plant extract & Euphorbia hirta & $\begin{array}{c}\text { Slowing of respiratory } \\
\text { movements, Reduction of } \\
\text { gastrointestinal motility }\end{array}$ & {$[6,7]$} \\
\hline $\begin{array}{l}\text { Administration of plant extract, then } \\
\text { acetic acid }\end{array}$ & Brugmansia suaveolens & Inhibition of abdominal cramps & [8] \\
\hline \multicolumn{4}{|l|}{ Ex vivo } \\
\hline \multirow{5}{*}{$\begin{array}{l}\text { Tracheal precontraction induced by } \\
\text { carbachol or acetylcholine then } \\
\text { application of increasing doses of } \\
\text { plant extract. }\end{array}$} & Dichrostachys cinerea & \multirow{13}{*}{ Smooth muscle relaxation } & [9] \\
\hline & Baphia nitida & & \\
\hline & Cassia occidentalis & & \\
\hline & Desmodium adscendens & & \\
\hline & Boerhavia diffusa & & \\
\hline \multirow{4}{*}{$\begin{array}{l}\text { Preliminary impregnation of } \\
\text { isolated trachea with the plant } \\
\text { extract, then stimulation by } \\
\text { histamine or acetylcholine. }\end{array}$} & Datura stramonium & & \\
\hline & Mentha pulegium & & {$[10]$} \\
\hline & Gossypium barbadense & & [11] \\
\hline & Guiera senegalensis & & {$[12]$} \\
\hline \multirow{3}{*}{$\begin{array}{l}\text { Precontraction of the trachea with } \\
\text { acetylcholine or histamine, then } \\
\text { application of increasing doses of } \\
\text { plant extract. }\end{array}$} & Foeniculum vulgare & & \\
\hline & Ammi visnaga & & [13] \\
\hline & Rosmarinus officinalis & & [14] \\
\hline $\begin{array}{l}\text { Precontraction of the trachea with } \\
\text { acetylcholine, potassium chloride or } \\
\text { histamine, then application of } \\
\text { increasing doses of plant extract. }\end{array}$ & Glycyrrhiza glabra & & {$[15,16]$} \\
\hline
\end{tabular}


Precontraction of the trachea induced by acetylcholine, then application of increasing doses of plant extract.

Waltheria indica

Contraction of guinea pig intestinal

smooth muscle induced by acetylcholine or barium chloride, then application of plant extract.

\begin{tabular}{|c|} 
\\
\\
Waltheria indica \\
\hline \\
Tamarindus indica
\end{tabular}

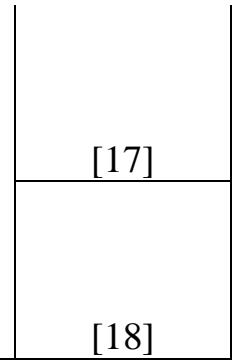

Table 3: Experimental protocols for highlighting anti-inflammatory activity.

\begin{tabular}{|c|c|c|c|}
\hline Methods & Plants & Results & References \\
\hline \multicolumn{4}{|c|}{ In vivo } \\
\hline $\begin{array}{l}\text { Administration of plant lectin in murine air } \\
\text { pouch model }\end{array}$ & & $\begin{array}{l}\text { Inhibition of cell } \\
\text { recruitment }\end{array}$ & [19] \\
\hline $\begin{array}{c}\text { Administration of plant extract in } \\
\text { carrageenan-induced rat paw edema model }\end{array}$ & Viscum album & $\begin{array}{c}\text { IC50: } 0.617 \pm 0.017 \\
\mu \mathrm{g} / \mathrm{mL}\end{array}$ & [20] \\
\hline \multirow[b]{4}{*}{$\begin{array}{l}\text { Administration of plant extract in pre- } \\
\text { treatment in formaldehyde } 1 \% \text {-induced } \\
\text { guinea pig paw edema model }\end{array}$} & Aframomum melegueta & $\begin{array}{l}\text { Edema inhibition: } \\
90.42 \pm 0.22 \%\end{array}$ & \multirow[b]{4}{*}{ [21] } \\
\hline & Citrus aurantifolia & $\begin{array}{l}\text { Edema inhibition: } 98 \\
\quad \pm 0.57 \% \text {. }\end{array}$ & \\
\hline & Sterculia setigera & $\begin{array}{l}\text { Edema inhibition: } \\
80.83 \pm 0.38 \%\end{array}$ & \\
\hline & Eucalyptus camaldulensis & $\begin{array}{l}\text { Edema inhibition } \approx \\
\text { lysine } \\
\text { acetylsalicylate }\end{array}$ & \\
\hline $\begin{array}{l}\text { Administration of plant extract in } \\
\text { carrageenan-induced rat paw edema model. }\end{array}$ & Crataegus monogyna & $\begin{array}{l}\text { Edema inhibition: } \\
72.4 \% \\
\end{array}$ & [22] \\
\hline $\begin{array}{l}\text { Administration of plant extract in } \\
\text { carrageenan-induced guinea pig paw edema } \\
\text { model. }\end{array}$ & Melissa officinalis & $\begin{array}{l}\text { Edema inhibition: } \\
65.38 \% \text {. }\end{array}$ & [23] \\
\hline $\begin{array}{l}\text { Croton oil-induced ear edema and } \\
\text { carrageenan-induced paw edema in guinea } \\
\text { pig, then administration of plant extract. }\end{array}$ & Parkia biglobosa & Edema Reduction & [24] \\
\hline \multirow{2}{*}{$\begin{array}{l}\text { Administration of plant extract in pre- } \\
\text { treatment in formaldehyde } 1 \% \text {-induced } \\
\text { guinea pig paw edema model }\end{array}$} & Lavandula angustifolia & \multirow[b]{2}{*}{ Edema inhibition } & \multirow[b]{2}{*}{ [25] } \\
\hline & Ocimum basilicum & & \\
\hline $\begin{array}{l}\text { Administration of plant extract in pre- } \\
\text { treatment in carrageenan or histamine- } \\
\text { induced guinea pig paw edema model }\end{array}$ & Sterculia tragacantha & Edema inhibition & [26] \\
\hline \multirow{2}{*}{$\begin{array}{l}\text { Carrageenan or formaldehyde-induced paw } \\
\text { edema, then administration of plant extract. }\end{array}$} & Euphorbia hirta & \multirow{2}{*}{$\begin{array}{l}\text { Dose-dependent } \\
\text { reduction of edema }\end{array}$} & [27] \\
\hline & Zingiber officinale & & [28] \\
\hline $\begin{array}{l}\text { Carrageenan or formaldehyde-induced paw } \\
\text { edema in guinea pig, then administration of } \\
\text { plant extract. }\end{array}$ & Adhatoda vasica & $\begin{array}{l}\text { Edema inhibition: } \\
59.51 \% \text {. }\end{array}$ & [5] \\
\hline \multicolumn{4}{|c|}{ In vitro } \\
\hline \multirow{2}{*}{$\begin{array}{l}\text { Oxidative stress model and measurement of } \\
\text { the trapping effect of the plant extract on } \\
\text { hydrogen peroxide and hypochlorous acid }\end{array}$} & Alchornea cordiforlia & $\begin{array}{l}\text { IC50: } 6.5 \mathrm{mg} / \mathrm{mL} \text { for } \\
\mathrm{H}_{2} \mathrm{O}_{2}\end{array}$ & \multirow[b]{2}{*}{ [29] } \\
\hline & Baphia nitida & $\begin{array}{l}\text { IC50: } 1.7 \mathrm{mg} / \mathrm{mL} \text { for } \\
\mathrm{HOCl}\end{array}$ & \\
\hline
\end{tabular}




\begin{tabular}{|c|c|c|c|}
\hline & Cassia occidentalis & $\begin{array}{c}\text { IC50: } 1.8 \mathrm{mg} / \mathrm{mL} \text { for } \\
\mathrm{HOCl}\end{array}$ & \\
\hline & Boerhavia diffusa & $\begin{array}{c}\text { IC50: } 6.8 \mathrm{mg} / \mathrm{mL} \text { for } \\
\qquad \mathrm{H}_{2} \mathrm{O}_{2}\end{array}$ & \\
\hline $\begin{array}{c}\text { Murine macrophages activated by } \\
\text { lipopolysaccharide }\end{array}$ & Viscum album & $\begin{array}{c}\text { IC50: } 70.20 \pm 1.094 \\
\mu \mathrm{g} / \mathrm{mL}\end{array}$ & [20] \\
\hline $\begin{array}{l}\text { Incubation with gastric and intestinal } \\
\text { physiological fluids }\end{array}$ & Mentha spicata & $\begin{array}{l}\text { Inhibition of } \\
\text { lipopolysaccharide- } \\
\text { induced PGE2 }\end{array}$ & [30] \\
\hline \multirow[b]{2}{*}{$\begin{array}{c}\text { Involving plant extract and serum albumin } \\
\text { solution }\end{array}$} & Salvia officinalis & $\begin{array}{l}\text { Inhibition of albumin } \\
\text { denaturation: } \\
99.18 \% \text {. }\end{array}$ & [31] \\
\hline & Thymus vulgaris & $\begin{array}{c}\text { Inhibition of albumin } \\
\text { denaturation: } 66.12 \pm \\
12.5 \% \text {. }\end{array}$ & [32] \\
\hline $\begin{array}{l}\text { Addition of the plant extract to a pro- } \\
\text { inflammatory enzyme solution } \\
\text { (Phospholipase A2 and 5-Lipoxygenase) }\end{array}$ & Waltheria indica & $\begin{array}{l}\text { Inhibition of enzyme } \\
\text { activity: } 60-80 \% \text {. }\end{array}$ & [17] \\
\hline
\end{tabular}

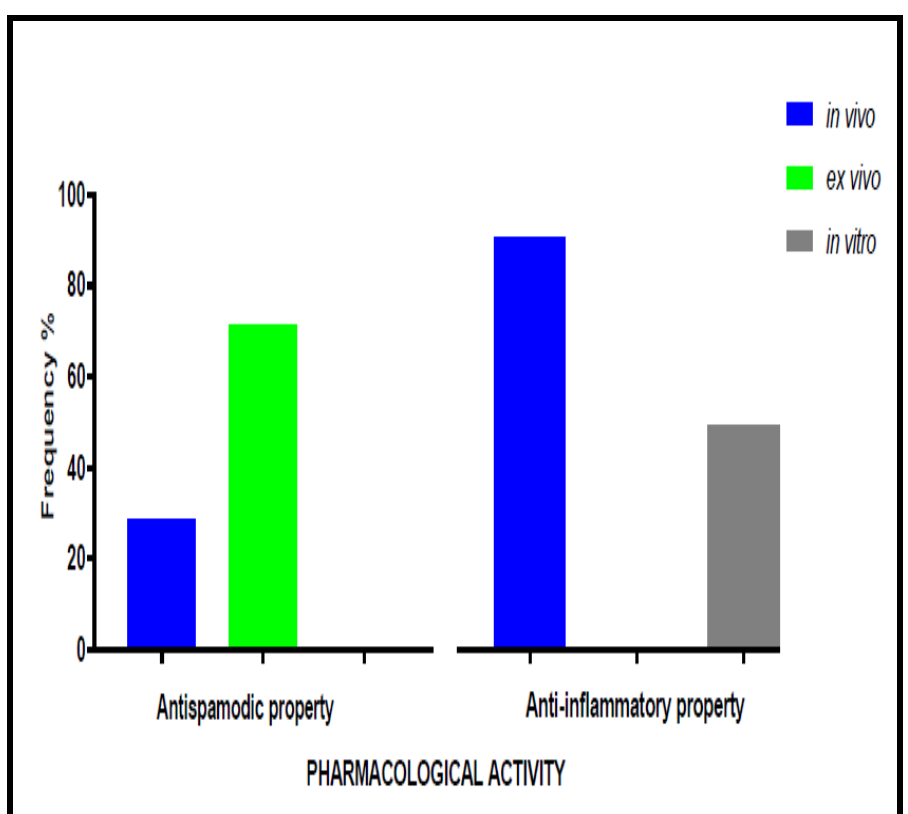

Figure 2: Implementation of pharmacological tests.

\section{Discussion}

The aim of this study was to analyse pharmacological evaluation data for traditional anti-asthmatic plants in Africa. The literature review identified 184 plant species used in the treatment of asthma in African traditional medicine. This great diversity of species used for the treatment of asthma in Africa is an indication of the importance of medicinal plants but also of the fact that asthma is an important health problem in Africa $[33,34]$.
Only 34 plant species, the majority of which are aromatic plants from the Mediterranean region of North Africa, have undergone preclinical pharmacological studies. These works consisted mainly in the identification of antispasmodic or spasmolytic and/or anti-inflammatory properties. Indeed, the pathophysiology of asthma is mainly characterized by bronchospasm and airway inflammation [35]. Consequently, the pharmacological management of asthma disease is carried out in conventional medicine by bronchodilator agents associated with antiinflammatory molecules, in particular corticosteroids [36]. Thus, it seems appropriate that the scientific valuation of anti-asthmatic medicinal plants provides evidence of antispasmodic or spasmolytic activity in favor of bronchospasm blockage on the one hand, and/or anti-inflammatory activity on the other.

The protocols for the identification of the activities of these plants against bronchospasm were based mainly on the inhibition of airways smooth muscles contraction previously induced. This corresponds to a curative treatment of the bronchoconstriction of asthma attack. Indeed, short-acting bronchodilators, without antiinflammatory effect, are recommended to release bronchial smooth muscle [37] because the usual forms, aerosols and dry powders or solutions for nebulization, by inhalation, increase mucociliary clearance and rapidly reach the smooth muscle of trachea and bronchi [38].

Experiments for highlighting anti-inflammatory property of anti-asthmatic plants mainly pre-treated 
laboratory animals with plant extracts, then induced edema by a phlogogenic agent. This approach corresponds to a preventive treatment of inflammation, in accordance with the basic treatment of asthma by inhaled glucocorticoids [37]. Indeed, asthma attacks most often result from the activation of cells of inflammation, and the production of various proinflammatory mediators [39].

\section{Conclusion}

The review of preclinical studies of medicinal plants used in the management of asthma in Africa showed that the African plant flora is rich and diverse. Very little evidence in animal experiments has been provided to enhance the properties claimed by traditional medicines. However, most of the experiments carried out were based on the physiopathology of asthma, on the one hand, by the demonstration of spasmolytic and/or anti-inflammatory effects, and on the other hand in accordance with curative bronchodilator treatment and anti-inflammatory preventive one. The results of this work justify the standardisation of experimental study protocols on the one hand, and the collaboration between scientists and practitioners of African traditional medicine on the other hand for the improvement of medicinal plants valorisation.

\section{Funding}

None declared.

\section{References}

1. El Mekki F, Taktak S, Mechaal S, et al. Asthma et pollution atmosphérique. Rev Pneumol Clin 2004; 60: 13-21.

2. Rochat T, Bridevaux P-O, Gerbase M, et al. Quel est le rôle de la pollution atmosphérique dans l'asthma? Rev Med Suisse 2012; 8: 2233-2236.

3. Jacquemot P. Les systèmes de santé en Afrique et l'inégalité face aux soins. Afr contemp 2012; 3: 9597.

4. OMS. Stratégie de l'OMS pour la médecine traditionnelle pour 2014-2023; 72p.

5. Kapgate SM, Patil AB. Adhatoda vasica: A critical review. Int J Green Pharm 2017; 11: S654-S662.

6. Chopra RN, Chopra IC, Handa KL et al. Indigenous drugs of India. Calcutta, India: Academic Publishers 1994.

7. Hore SK, Ahuja V, Mehta G et al. Effect of aqueous Euphorbia hirta leaf extract on gastrointestinal motility. Fitoterapia 2006; 77: 35-38.
8. Parker AG, Peraza GG, Sena $J$ et al. Antinociceptive effects of the aqueous extract of Brugmansia suaveolens flowers in mice. Biol Res Nurs 2007; 8: 234-239.

9. Irié-N'guessan G, Champy P, Kouakou-Siransy G et al. Tracheal relaxation of five Ivorian antiasthmatic plants: Role of epithelium and $\mathrm{K}+$ channels in the effect of the aqueous-alcoholic extract of Dichrostachys cinerea root bark. J Ethnopharmacol 2011; 138: 432-438.

10. Ullrich SF, Hagels H, Kayser O. Scopolamine: A journey from the field to clinics. Phytochem Rev 2017; 16: 333-353.

11. Soares PMG, Pires AF, de Souza EP et al. Relaxant effects of the essential oil of Mentha pulegium L. in rat isolated trachea and urinary bladder. J Pharm Pharmacol 2012; 64: 1777-1784.

12. Mbagnick BD, DSc thesis, Cheik Anta Diop University (Dakar, Senegal, 2011).

13. Badgujar SB, Patel VV, Bandivdekar AH. Foeniculum vulgare Mill: A Review of Its Botany, Phytochemistry, Pharmacology, Contemporary Application, and Toxicology. Biomed Res Int 2014; 1-32.

14. Goetz P. Les antispasmodiques en phytothérapie. Phytothérapie 2017; 15: 182-188.

15. Aqel MB. Relaxant effect of the volatile oil of Rosmarinus officinalis on tracheal smooth muscle. J Ethnopharmacol 1991; 33: 57-62.

16. Lui B. Isoliquiritigenin, a flavonoid from licorice, relaxes guinea-pig tracheal smooth muscle in vitro and in vivo: role of cGMP PKG pathway. Eur $\mathbf{J}$ Pharmacol 2008; 587: 257-66.

17. Zongo FER, DSc thesis, University of Grenoble (Grenoble, France, 2013).

18. Ouédraogo S, Kini F, Coulibaly-Traoré M, et al. Etudes des propriétés pharmacologiques du fruit de Tamarindus Indica Linn, Utilisé comme Laxatif et Antipaludique en Médecine Traditionnelle. Fruit, Veg Cereal Sci Biotech 2010; 4: 31-35.

19. Lavastre V, DSc thesis, University of Quebec (Quebec, Canada, 2009).

20. Murthuza S, Manjunatha BK. In vitro and in vivo evaluation of anti-inflammatory potency of Mesua ferrea, Saraca asoca, Viscum album \& Anthocephalus cadamba in murine macrophages raw 264.7 cell lines and Wistar albino rats. BeniSuef Univ J Basic Appl Sci 2018; 7: 719-723.

21. Gbenou JD, Ahounou JF, Akakpo HB. Phytochemical composition of Cymbopogon citratus and Eucalyptus citriodora essential oils and their anti-inflammatory and analgesic properties on Wistar rats. Mol Biol Rep 2013; 2: 1127-1134. 
22. Nabavi SF, Habtemariam S, Ahmed $T$ et al. Polyphenolic composition of Crataegus monogyna Jacq. From chemistry to medical applications. Nutriments 2015; 7: 7708-7728.

23. Bounihi A, MSc thesis, Mohammed V University (Casablanca, Morroco, 2015).

24. Nwaehujor CO, Ezeigbo II, Udeh NE, et al. Antiinflammatory and anti-oxydant activities of the methanolic extracts of the stalk of Parkia biglobosa (Jacq) Benth. Hygeia J D Med 2011; 34-40.

25. Nouzha K, Nihad FI, MSc thesis, Mentouri Brothers University (Constantine, Egypt, 2018).

26. Mogbojuri OM, Adedapo AA, Abatan M. Phytochemical screening, safety evaluation, antiinflammatory and analgesic studies of the leaf extracts of Sterculia tragacantha. J Complement Integr Med 2016; 13: 221-228.

27. Lanhers MC, Fleurentin J, Dorfman P. Analgesic, antipyretic and anti-inflammatory properties of Euphorbia hirta L. Planta Med 1991; 57: 225-231.

28. Jeena K, Liju VB, Kuttan R. Antoxidant, antiinflammatory and antinociceptive activities of essential oil from ginger. Indian $\mathbf{J}$ Physiol Pharmacol 2013; 57: 51-62.

29. Kouakou-Siransy G, Sahpaz S, Irié-Nguessan G, et al. Oxygen species scavenger activities and phenolic contents of four West African plants. Food chem 2010; 118: 430-435.

30. Pearson W, Fletcher RS, Kott LS et al. Protection against LPS-induced cartilage inflammation and degradation provided by a biological extract of Mentha spicata. BMC Complement Med Ther 2010; 10: 1-11.
31. Taïba I, Boumahrat M, Boulifa A. MSc thesis, Mentouri Brothers University (Constantine, Egypt, 2017).

32. Khaldi FZ, MSc thesis, Mentouri Brothers University (Constantine, Egypt, 2015).

33. Helpern M.G. Sinusitis and asthma association, influence and principle of management. Allergol Immunopath 1995; 23.

34. Ndri-Yoman A. T. Plan d'action intégré de prévention et de prise en charge des maladies chroniques non transmissibles en Côte d'Ivoire 2011-2014. République de Côte d'Ivoire-Ministère de la Santé et de la lutte contre le SIDA. 30p.

35. Bousquet J, Jeffery PK, Busse WW, et al. Asthma from bronchoconstriction to airways inflammation and remodeling. Am J Respir Crit Care Med 2000; 161: 1720-1745.

36. Chung KF, Caramori G, Adcock IM. Inhaled corticosteroids as combination therapy with betaadrenergic agonists in airways disease: Present and future. Eur J Clin Pharmacol 2009; 65: 853-871.

37. Global Strategy for Asthma Management and Prevention, Global Initiative for Asthma (GINA). 2012.

38. Société Ivoirienne de Pneumo-Phtisiologie (SIPP). Consensus national sur la prise en charge de l'asthma en Côte d'Ivoire. 1ère édition, 2005; 49 p.

39. Pfeister A, Gaultier C, Jaubert F et al. Physiologie \& physiopathologie immuno-allergologique de l'appareil respiratoire. Académie de Paris: Université René Descartes, facultés de médecine Cohin, Paris, 1995.

This manuscript was peer-reviewed

Mode of Review: Single-blinded

Academic Editor: Mr. Kamran Ahmad

Copyright: C2020 N'guessan-Irié GA, et al. This article is distributed under the terms of the Creative Commons Attribution 4.0 International License (http://creativecommons.org/licenses/by/4.0/), which permits unrestricted use, distribution, and reproduction in any medium, provided you give appropriate credit to the original author(s) and the source, provide a link to the Creative Commons license, and indicate if changes were made. 